\title{
Surgical aortic valve replacement due to infective endocarditis after transcatheter aortic valve implantation with the self-expanding Portico valve prosthesis
}

\author{
Yukiharu Sugimura, Shintaro Katahira, Rene Lopez Lopez, Philipp Rellecke, Artur Lichtenberg, \\ Payam Akhyari \\ Department of Cardiovascular Surgery, Medical Faculty, Heinrich Heine University, Düsseldorf, Germany \\ Correspondence to: Univ.-Prof. Dr. med. Payam Akhyari. Department of Cardiovascular Surgery, Heinrich-Heine-University, Medical Faculty, \\ Moorenstr 5, Düsseldorf 40225, Germany. Email: payam.akhyari@med.uni-duesseldorf.de.
}

Submitted Mar 28, 2019. Accepted for publication Apr 17, 2019.

doi: 10.21037/acs.2019.05.07

View this article at: http://dx.doi.org/10.21037/acs.2019.05.07

\section{Clinical vignette}

An 83-year-old man with coronary heart disease, previous pacemaker implantation due to Mobitz II atrioventricular block, hypertension, hyperuricemia and dementia, presented with dyspnea NYHA III+. Transcatheter aortic valve implantation (TAVI) had been performed 4 months before current admission in another hospital using Portico $27 \mathrm{~mm}$ prosthesis (introduced by St. Jude Medical, Minneapolis, Minnesota, today Abbott), where grade II aortic valve regurgitation and paravalvular leakage (PVL) had remained, despite two additional post-dilatations. Despite initial freedom from symptoms, the patient presented at our emergency department with acute cardiac decompensation. Laboratory workup revealed increased inflammatory parameters and blood culture results which were positive for Staphylococcus aureus. Transthoracic and transesophageal echocardiography showed severely impaired systolic left ventricular function with global hypokinesia (ejection fraction; $35 \%$ ) and high-grade paravalvular aortic valve regurgitation, as well as de novo manifested transvalvular jet. We performed surgical aortic valve replacement (SAVR) because of prosthetic valve endocarditis (PVE).

\section{Surgical techniques}

\section{Preparation}

Recompensation therapy accompanied by definitive antibiotic therapy was initiated to prepare for a surgical procedure.

\section{Exposition}

Under general anesthesia, the median sternotomy with central cannulation for extracorporeal circulation was performed. Cardiac arrest and myocardial protection were achieved via retrograde cardioplegia using cold blood retro-cardioplegia.

\section{Operation}

At first, pacemaker leads were removed, displaying small amounts of suspicious tissue covering the atrial segments. The Portico valve with its sizeable supravalvular stent presented as partially grown into the intima of the aorta. The ascending aorta could be preserved with a tedious preparation of the TAVI stent from the aortic wall, although the integrity of an intimal layer of the aortic wall adhering to the TAVI prosthesis had to be sacrificed. The native aortic valve cusps were strongly fused with the pericardial skirt portion of the TAVI prosthesis. The non-coronary sinus of Valsalva could not be preserved due to relevant calcification. The intraoperative macroscopic inspection revealed no clear vegetation on the TAVI prosthesis. A 23-mm biological valve (Edwards Perimount Magna Ease) was implanted in a supra-annular position. The noncoronary sinus of Valsalva was replaced with Dacron. At last, an epicardial pacemaker system was implanted.

\section{Completion}

The patient was transferred to the intensive care unit with 
moderate catecholamine support, which was weaned in the following 48 hours. The patient was extubated on the next day, and levosimendan was intravenously over 24 hours for further stabilization.

\section{Comments}

\section{Clinical results}

Portico is a self-expanding repositionable and retrievable transcatheter aortic valve prosthesis, with a low incidence of PVL reported in the literature $(1,2)$.

PVE is a severe complication of TAVI. According to recently published results from an international multicenter registry with 7,499 patients, the one-year incidence of PVE was $0.05 \%(n=53)$, however, with an inhospital mortality rate of $47.2 \%$ (3). The necessity of oral intubation, as well as PVE affecting a self-expandable valve system, were the main risk factors for mortality. A new valve intervention was performed in a minority of $11 \%$ of all PVE patients $(\mathrm{n}=6)$. Interestingly, SAVR was performed in only 3 patients, of which one patient died during hospitalization. A similar trend is reported from another international registry with most patients presenting with PVE after TAVI (82\%) being treated conservatively with antibiotics and only a few (15\%) undergoing surgery (4). Overall, in-hospital mortality was $36 \%$. Upon multivariate analysis, age [hazard ration (HR): 0.97, 95\% CI: 0.94-0.99], male gender (HR: 1.69, 95\% CI: 1.1-2.52), diabetes mellitus (HR: 1.52, 95\% CI: 1.02-2.29) and the presence of moderate or higher grade PVL (HR: 2.05, 95\% CI: 1.28-3.28) were predisposing factors for PVE. The surgical intervention did not influence the risk of mortality in this study. Collectively, SAVR for TAVI-PVE is occasionally considered in patients with uncontrolled infection or refractory heart failure, overgrown vegetation or abscess, aneurysmal formation or thromboembolic events (5).

\section{Advantage}

SAVR is a definitively reliable procedure in PVE. A few cases of valve-in-valve procedure have been reported as an alternative to SAVR (3). It is, however, questionable whether the infectious status can be improved in a sustained manner by a valve-in-valve procedure and the risk of recurrence of PVE will likely remain somewhat unfavorable with infected prosthetic material left in-situ, although reliable data on this topic is missing.
From another point of view, preparing a safe route for controlled application of the cardioplegic solution is of particular importance in this operation since access to coronary ostia for controlled perfusion and secure myocardial protection is limited by two factors: (I) size of the stent cells and calcified native cusps displaced towards the sinuses of Valsalva, limiting the surgeon's possibilities for direct cannulation of the coronary ostia; (II) moderate or severe aortic regurgitation often present in PVE limits the efficacy of cardioplegia administered via the ascending aorta. In our opinion, bicaval cannulation and direct perfusion of the coronary sinus is favored by us and many other surgeons, this strategy has been proven successful in the present case with preoperatively severely depressed LV function.

\section{Caveats}

SAVR post-TAVI due to PVE represents a technically demanding procedure with elevated perioperative risk. Considering the design of this self-expanding prosthesis, the large stent crown represents a technical challenge for surgical removal. Occasionally, root replacement may become necessary, which considerably increases the overall risk of the procedure.

\section{Acknowledgments}

None.

\section{Footnote}

Conflicts of Interest: The authors have no conflicts of interest to declare.

\section{References}

1. Denegri A, Nietlispach F, Kottwitz J, et al. Real-world procedural and 30-day outcome using the Portico transcatheter aortic valve prosthesis: A large single center cohort. Int J Cardiol 2018;253:40-4.

2. Mas-Peiro S, Vasa-Nicotera M, Weiler H, et al. ThirtyDay Outcomes in 100 Consecutive Patients Undergoing Transfemoral Aortic Valve Replacement With the Portico Valve on an All-Comer Basis. J Invasive Cardiol 2017;29:431-6.

3. Amat-Santos IJ, Messika-Zeitoun D, Eltchaninoff H, et al. Infective endocarditis after transcatheter aortic valve 
implantation: results from a large multicenter registry. Circulation 2015;131:1566-74.

4. Regueiro A, Linke A, Latib A, et al. Association Between Transcatheter Aortic Valve Replacement and Subsequent Infective Endocarditis and In-Hospital Death. JAMA

Cite this article as: Sugimura Y, Katahira S, Lopez Lopez R, Rellecke P, Lichtenberg A, Akhyari P. Surgical aortic valve replacement due to infective endocarditis after transcatheter aortic valve implantation with the self-expanding Portico valve prosthesis. Ann Cardiothorac Surg 2019;8(6):699-701. doi: 10.21037/acs.2019.05.07
2016;316:1083-92.

5. Ahmad K, Klaaborg KE, Hjortdal V, et al. Prosthetic valve endocarditis after transcatheter aortic valve implantationdiagnostic and surgical considerations. J Thorac Dis 2016;8:E1213-8. 